\title{
On the Robustness of Two-Phase Flow Models for Bubbly Flows
}

\author{
Henrik Ström ${ }^{1}$ and Dia Zeidan ${ }^{2}$ \\ ${ }^{1}$ Department of Applied Mechanics, Chalmers University of Technology, SE-412 96 Gothenburg, Sweden \\ ${ }^{2}$ School of Basic Sciences and Humanities, German Jordanian University, Amman, Jordan
}

\begin{abstract}
Lack of robustness, manifesting as an inability to suppress the growth of numerical round-off errors, is a well-known challenge with many conventional two-fluid models. In the current work, a hyperbolic two-phase mixture model in conservative form is analysed and assessed in relation to a single-pressure two-fluid model. The robustness of the simulation results indicates that the mixture model and the associated numerical methods may be well suited to enable high-resolution simulations of more complex two-phase phenomena. In particular, significant advantages in terms of stability and robustness of the mixture model formulation are found in comparison to the non-hyperbolic and non-conservative single-pressure two-fluid model.
\end{abstract}

Keywords: two-phase flow, bubbly flow, stability, robustness.

PACS: 47.55.-t, 47.55.dd, 02.60.Lj, 02.60.-x, 47.55.-t

\section{INTRODUCTION}

Gas-liquid multiphase flows form the basis for a huge number of industrial processes. Because of the large sizes of conventional reactor, separation and transportation systems, the two-phase phenomena within such equipment typically have to be investigated with averaged descriptions - the so-called two-fluid models. Such models are derived from conservation laws for each phase for mass, momentum and energy, where phase interactions are accounted through interfacial exchange terms. Two main issues of concern are related to these models in their standard form: the occurrence of complex eigenvalues (resulting in ill-posed or non-hyperbolic initial-boundary-value problems) and the non-conservative temporal and spatial terms appearing in their final formulation. Lack of hyperbolicity and conservativity characters of such models results in spurious oscillations in the numerical resolutions which render high-fidelity simulations of small-scale two-phase phenomena in industrial processes intractable [1,2].

The purpose of this paper is to take a first step in the direction of applying a recently developed two-phase flow model based on the theory of thermodynamically compatible systems of hyperbolic conservation laws to the analysis of realistic process equipment. The model is formulated in terms of parameters of state for the mixture and describes gas and liquid phases in total non-equilibrium. Further, the model consists of a mixture mass, mixture momentum and a balance law for the relative velocity between the two phases. The model is implemented into a well-known commercial computational fluid dynamics (CFD) code, namely ANSYS Fluent. The ability of the model to suppress the growth of numerical round-off errors is assessed in relation to a conventional, non-hyperbolic and non-conservative single-pressure two-fluid model. The model equations along with the numerical treatment are presented briefly in the following sections.

\section{Mixture model}

The current two-phase mixture model is formulated based on the assumption that local equilibrium between the phases is attained over short spatial scales, so that the mixture parameters of state [3,4] may be introduced and the model becomes:

$$
\frac{\partial}{\partial t}(\rho)+\frac{\partial}{\partial x}(\rho u)=0
$$

International Conference of Numerical Analysis and Applied Mathematics (ICNAAM 2016)

AIP Conf. Proc. 1863, 030031-1-030031-4; doi: 10.1063/1.4992184

Published by AIP Publishing. 978-0-7354-1538-6/\$30.00 


$$
\begin{gathered}
\frac{\partial}{\partial t}(\rho u)+\frac{\partial}{\partial x}\left(\rho u^{2}+P+\rho c(1-c) u_{r}^{2}\right)=\mathcal{S}, \\
\frac{\partial}{\partial t}\left(u_{r}\right)+\frac{\partial}{\partial x}\left(u u_{r}+(1-2 c) \frac{u_{r}^{2}}{2}+\psi(P)\right)=\pi .
\end{gathered}
$$

The symbols in the equations presented above have the following meanings: $t$ is time and $x$ is spatial position, $\rho$, $u, P, c$ and $u_{r}$ denote, respectively, the mixture density, mixture velocity, mixture pressure, gas mass void fraction and relative velocity between the two phase systems. It is assumed here that the flow is isentropic that no mass transfer occurs between the phases. Furthermore, the basic physics is that the two phases are coupled by the relative velocity equation rather than the phase momentum equations. In this regard, additional terms arise in the framework of velocity non-equilibrium behaviour showing the benefits of using mixture formulations. Based on such formulations, the term $\psi(P)$ is a function that depends on phase equations of state. A more detailed description of all particular analysis involved in the well-posedness of this model, including the form adopted for $\psi(P)$, can be found in [4]. The terms $\mathcal{S}$ and $\pi$ are source terms owing to the interphase exchange processes, and are not considered further in the current work.

The model is implemented and solved in a one-dimensional in-house code using a total variation diminishing (TVD) slope limiter centre (SLIC) numerical scheme. The TVD SLIC is a Godunov centred-type scheme for hyperbolic conservation laws that achieves second-order accuracy by using MUSCL extrapolation [5]. In addition, the model is also implemented in the commercial CFD-software ANSYS Fluent 15.0.7, where it is complemented by an advection equation for the volume fraction of the secondary phase:

$$
\frac{\partial}{\partial t}\left(\alpha \rho_{2}\right)+\frac{\partial}{\partial x}\left(\alpha \rho_{2} u_{2}\right)=0 .
$$

The Fluent implementation uses a segregated solver based on the Semi-Implicit Method for Pressure-Linked Equations (SIMPLE) algorithm [6], with first-order implicit time stepping, first-order upwind discretization of the convective terms and the Pressure Staggering Option (PRESTO!) scheme for interpolation of the face pressures. Finally, a single-pressure two-fluid model available in Fluent is also employed in the assessment of the performance of the mixture two-phase model developed here. This model consists of Equation 4 together with the condition $\alpha_{1}=1-\alpha_{2}$ and separate momentum balance equations for the two phases $(i=1,2)$ :

$$
\rho_{i}\left[\frac{\partial}{\partial t}\left(\alpha_{i} u_{i}\right)+\frac{\partial}{\partial x}\left(\alpha_{i} u_{i}^{2}\right)\right]=-\alpha_{i} \frac{\partial}{\partial x} P+\mu_{i} \frac{\partial}{\partial x}\left(\alpha_{i} \frac{\partial u_{i}}{\partial x}\right)+\alpha_{i} \rho_{i} g+M_{i} .
$$

The numerical solution procedure is the same as for the mixture model, with second-order central differencing of the additional second term on the right-hand side of Equation 5. The momentum interface exchange term $M_{i}$ accounts for drag only [7].

\section{Test cases}

Two types of simulations are considered: the first is an expansion tube test problem of [4], and the second one is an instability growth test of [2]. The expansion tube test problem is performed for a one-dimensional computational domain of $[-10,10]$ with the initial conditions $\left(\rho, u, u_{r}\right)=[800,-100,0]$ for $-10 \leq x \leq 0$ and $\left(\rho, u, u_{r}\right)=[800,100,0]$ for $0<x \leq 10$. The mesh resolution is 100 grid points in the $x$-direction. The aim of this simulation is to assess the implementation of the current mixture model into the Fluent code. The equations of state used for the gas and liquid correspond to those of a compressible, isothermal ideal gas and an incompressible liquid, respectively.

Next, the instability growth problem is performed for a two-dimensional periodic domain of $[0,0.4]$ in the periodic $(x)$ direction and $[0,0.1]$ in the other direction (free-slip walls). In the instability growth test, a previously published single-pressure two-fluid model setup [2] is also used as a reference. The initial condition is $\left(u, u_{r}, P, \alpha\right)=[0,0,0,0.1]$ in the entire domain. The aim of this simulation is to elucidate the rate at which the numerical model allows small numerical round-off errors to grow, or whether such errors may be suppressed entirely. The mesh resolution is 100 grid points in the $x$-direction and one grid point in the $y$-direction. Due to the lack of detailed resolution in the $y$-direction, the simulation is essentially one-dimensional. The existence of a second spatial dimension is however important for the possibility to assess the development of instabilities in a two-fluid model. The boundary conditions in the $x$-direction are periodic (with a prescribed pressure jump) and the upper and lower sides in the $y$-direction have free-slip conditions. Both phases are assumed to be incompressible and isothermal.

The emergence of instabilities is quantified via a global, time-resolved uniformity index [2]: 


$$
\Phi(t)=\frac{\alpha_{\max }-\alpha_{\min }}{\alpha_{\text {avg }}}
$$

Here, $\alpha_{\max }$ is the maximum volume fraction of the secondary phase in any cell at time $t$, and $\alpha_{\min }$ and $\alpha_{a v g}$ are the corresponding minimum and average values. A uniform $\alpha$-field is thus characterized by $\Phi=0$, whereas non-zero values correspond to the emergence of instabilities. $\Phi=1 / \alpha_{a v g}$ is a theoretical upper limit corresponding to fully separated flow.

\section{SIMULATION RESULTS}

\section{Expansion tube test}

The predictions for the two codes for the expansion tube test problem are compared in Figure 1. The overall agreement is acceptable, with some noticeable deviations for the magnitude of the relative velocity. It may be concluded that the addition of Equation 4 to the model specification, along with the different numerical procedure to solve the complete set of equations, has an effect on the predicted behaviour, but that the discrepancies are only quantitative. No adverse effects on stability are observed for this test case.
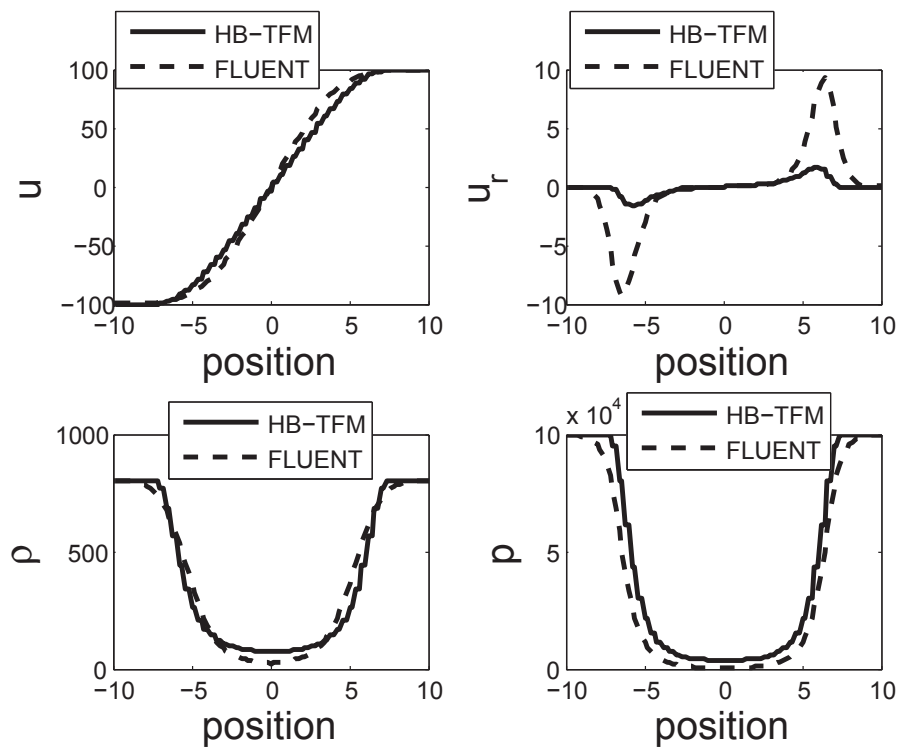

FIGURE 1. Code comparison for the expansion tube test problem: mixture velocity $(u)$, relative velocity $\left(u_{r}\right)$, mixture density $(\rho)$, and pressure $(P)$.

\section{Instability growth test}

The results from the instability growth test are shown in Figure 2, from which several important inferences can be made. Firstly, the full two-fluid model exhibits a marked growth of small-scale noise in the volume fraction field, as characterized by the increasing values of $\Phi$. It is known from before that, due to the ill-posedness of the model equations, this type of model is prone to display such types of instability growth [2]. In comparison, the herein presented mixture two-phase model only gives rise to minuscule $\Phi$-values of the same order of magnitude as the numerical uncertainty in the double-precision solver. The result from the first instability growth test is therefore that the mixture formulation is superior to the full two-fluid model in terms of suppressing the growth of small numerical errors. It is noticeable that the conclusion holds both for the case when the complete model is evaluated but no relative 

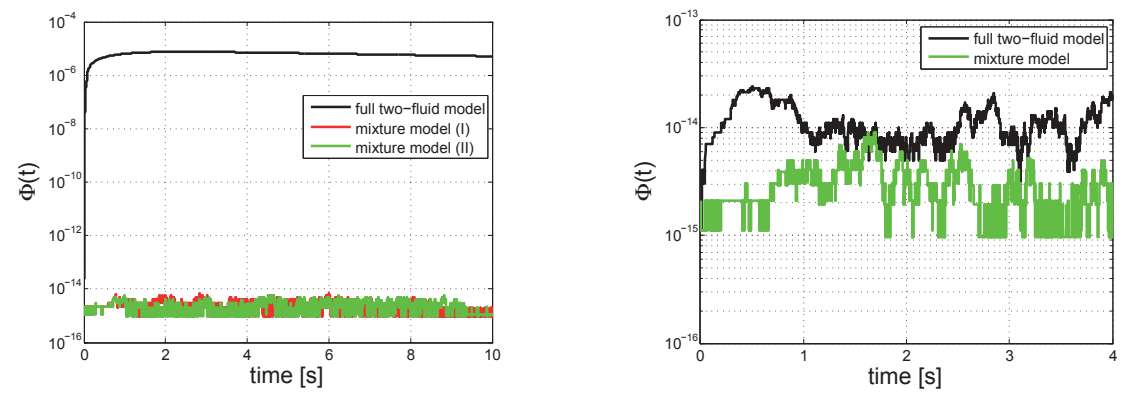

FIGURE 2. Temporal evolution of the non-uniformity index $\Phi$ in the instability growth tests. Left: Results from the full two-fluid model are compared with the mixture model for the case of no relative velocity (I) and a constant relative velocity equal to that observed in the full two-fluid model (II). Right: Results from the full two-fluid model are compared with the mixture model for a case where $\rho_{1}=\rho_{2}$.

velocity develops (case I in Figure 2) and for the case where the relative velocity is fixed to the same value as obtained in the full two-fluid model simulations (case II in the same figure).

In the full two-fluid model, the phase velocities are coupled via the momentum exchange term $M_{i}$ in Equation 5 , whereas in the hyperbolic mixture model, the coupling occurs via the evolution of the relative velocity. As the coupling between the phases is accounted for differently in the two types of model, an additional instability growth test is also performed for a case in which there is no physical reason for a relative velocity to manifest. The appearance of non-negligible $\Phi$-values in such a test points to an underlying unstable model character, and can therefore serve as a powerful basis for a relative assessment of the two model formulations.

The result of the second instability growth test is shown to the right in Figure 2. As this test is performed for identical phase densities, it allows for a direct comparison of the results - any non-dampened instability is now a true reflection of improper underlying model characteristics. It can be seen that the current mixture model still shows nonuniformities on the order of the numerical accuracy only, whereas the full two-fluid model exhibits a faster and more pronounced growth of the instabilities. For the initial time period studied, the latter model displays $\Phi$-values that are at least an order of magnitude larger than those of the mixture model, supporting the conclusion that the mixture model formulation could offer significant advantages over the conventional model for studies of small-scale instabilities in bubbly gas-liquid flows.

\section{CONCLUSIONS}

A hyperbolic two-phase mixture model in conservative form is presented and its implementation into a commercial CFD code is outlined. The model formulation accounts for the mechanical and velocity non-equilibrium between the two phases. The model and the numerical methods applied in the solution procedure have been shown to be computationally efficient and robust, the main advantage being that the model equations are solved using mixture formulations. The robustness of the simulation results indicates that the mathematical model and the associated numerical method may be extended in various ways to address more complex phenomena. In particular, an evaluation of the performance of the mixture model to that of a single-pressure two-fluid for bubbly gas-liquid flows indicates significant advantages in terms of stability and robustness of the model formulation.

\section{REFERENCES}

[1] T. Dinh, R. Nourgaliev, T. Theofanous, Proc. NURETH03, 2003.

[2] H. Ström, S. Sasic, K. Jareteg \& C. Demazière, Int. J. Chem. React. Eng. 13, 449-459, 2015.

[3] E. Romenski, A. D. Resnyansky, E. F. Toro, Quart. Appl. Math. 65, 259-279, 2007.

[4] D. Zeidan, Appl. Math. Comput. 272, 707-719, 2016.

[5] E.F. Toro, Riemann Solvers and Numerical Methods for Fluid Dynamics: A Practical Introduction, (3rd ed.), Springer, 2009.

[6] S. Patankar, Numerical Heat Transfer and Fluid Flow, Taylor \& Francis, 1980.

[7] L. Schiller, Z. Naumann, Zeitschrift des Vereines Deutscher Ingenieure, 1935. 